\title{
The Adomian Decomposition Method for Solving Nonlinear Partial Differential Equation Using Maple
}

\author{
Dalal Adnan Maturi, Honaida Mohammed Malaikah \\ Department of Mathematics, Faculty of Science, King Abdulaziz University, Jeddah, Saudi Arabia \\ Email: dmaturi@kau.edu.sa,hmalaikah@kau.edu.sa
}

How to cite this paper: Maturi, D.A. and Malaikah, H.M. (2021) The Adomian Decomposition Method for Solving Nonlinear Partial Differential Equation Using Maple. Advances in Pure Mathematics, 11, 595-603. https://doi.org/10.4236/apm.2021.116038

Received: May 3, 2021

Accepted: June 25, 2021

Published: June 28, 2021

Copyright $\odot 2021$ by author(s) and Scientific Research Publishing Inc. This work is licensed under the Creative Commons Attribution International License (CC BY 4.0).

http://creativecommons.org/licenses/by/4.0/

\begin{abstract}
The nonlinear partial differential equation is solved using the Adomian decomposition method (ADM) in this article. A number of examples have been provided to illustrate the numerical results, which is the comparison of the exact and numerical solutions, and it has been discovered through the tables that the amount of error between the exact and numerical solutions is very small and almost non-existent, and the graph also shows how the exact solution of absolutely applies to the numerical solution. This demonstrates the precision of the Adomian decomposition method (ADM) for solving the nonlinear partial differential equation with Maple18. And that in terms of obtaining numerical results, this approach is characterized by ease, speed, and high accuracy.
\end{abstract}

\section{Keywords}

Nonlinear Partial Differential Equation, Adomian Decomposition Method, Maple18

\section{Introduction}

The aim of this study is to use Maple18 to solve the Volterra-Fredholm integral equation with the Adomian decomposition process. Integral equations are fundamental sciences in our everyday lives; they describe physical, chemical, engineering, and medical phenomena, and they also help us find analytical and numerical solutions to these problems. 
A reliable Modification of Adomain Decomposition Method [1]. A new Modification of the Adomain Decomposition Method for Linear and Nonlinear Operators [2]. Lorenz equations are solved using a decomposition method [3]. Using Adomian's decomposition method to solve the Riccati differential equation [4].

Numeric-analytic integration of strongly nonlinear and chaotic oscillators using Adomian decomposition [5]. For fourth-order boundary value problems, the extended Adomian decomposition method [6]. The Adomian decomposition approach has been used to solve multipoint boundary value problems [7]. A new algorithm for evaluating Adomian polynomials has been developed [8]. An efficient algorithm for the multivariable Adomian polynomials [9]. Convenient analytic recurrence algorithms for the Adomian polynomials [10]. A review of the Adomian decomposition method and its applications to fractional differential equations are discussed in this paper [11]. A bibliography of the Adomian decomposition method's theory and applications [12]. Nonlinear integral equation solutions are more difficult to solve than linear integral equation solutions [13], and there are several analytical and computational methods for solving both linear and nonlinear integral equations [14]-[19].

MATLAB and Maple were used to implement the Adomian decomposition method for the Fredholm integral equation of the second kind. To solve the Fredholm integral equation of the second kind [20], the Adomian decomposition method was employed. In addition, using Maple, a Modified research approach for solving the Volterra integral equation of the second kind [21]. The Adomian Decomposition Method for Solving Volterra-Fredholm Integral Equation Using Maple [22].

In this article, we used the Maple algorithm to apply the Adomian decomposition method to various cases, such as finding the approximate solution, comparing it to the exact solution, and determining the amount of error between the approximate solution and the exact solution.

\section{Adomian Decomposition Method}

Consider the nonlinear partial differential equation given in an operator form

$$
L_{x} u(x, y)+L_{y} u(x, y)+R(u(x, y))+F(u(x, y))=g(x, y)
$$

where $L_{X}$ is the highest order differential in $X, L_{y}$ is the highest order differential in $y, R$ contains the remaining linear terms of lower derivatives, $F(u(x, y))$ is an analytic nonlinear term, and $g(x, y)$ is an inhomogeneous or forcing term.

Assuming that the operator $L_{x}$ meets the two bases of selection, therefore we set

$$
L_{x} u(x, y)=g(x, y)-L_{y} u(x, y)-R(u(x, y))-F(u(x, y))
$$

Applying $L_{x}^{-1}$ to both sides of (1) gives

$$
u(x, y)=\varnothing_{0}-L_{x}^{-1} g(x, y)-L_{x}^{-1} L_{y} u(x, y)-L_{x}^{-1} R(u(x, y))-L_{x}^{-1} R(u(x, y)),
$$




$$
\varnothing_{0}=\left\{\begin{array}{lr}
u(0, y) & \text { for } L=\frac{\partial}{\partial x} \\
u(0, y)+x u_{x}(0, y) & \text { for } L=\frac{\partial^{2}}{\partial x^{2}} \\
u(0, y)+x u_{x}(0, y)+\frac{1}{2 !} x^{2} u_{x x}(0, y) & \text { for } L=\frac{\partial^{3}}{\partial x^{3}} \\
u(0, y)+x u_{x}(0, y)+\frac{1}{2 !} x^{2} u_{x x}(0, y)+\frac{1}{3 !} x^{3} u_{x x x}(0, y) & \text { for } L=\frac{\partial^{4}}{\partial x^{4}}
\end{array}\right.
$$

We proceed in exactly the same manner by calculating the solution $u(x, y)$. In a series form

$$
u(x, y)=\sum_{n=0}^{\infty} u_{n}(x, y),
$$

And the nonlinear term

$$
F(u(x, y))=\sum_{n=0}^{\infty} A_{n},
$$

where $A_{n}$ are Adomian polynomials that can be generated for all forms of nonlinearity. Based on these assumptions, Equation (2) becomes

$$
\begin{aligned}
\sum_{n=0}^{\infty} u_{n}(x, y)= & \varnothing_{0}-L_{x}^{-1} g(x, y)-L_{x}^{-1} L_{y}\left(\sum_{n=0}^{\infty} u_{n}(x, y)\right) \\
& -L_{x}^{-1} R\left(\sum_{n=0}^{\infty} u_{n}(x, y)\right)-L_{x}^{-1}\left(\sum_{n=0}^{\infty} A_{n}\right),
\end{aligned}
$$

The components, $u_{n}(x, y), n \geq 0$ of the solution $u(x, y)$ can be recursively determined by using the relation

$$
\begin{gathered}
u_{0}(x, y)=\varnothing_{0}-L_{x}^{-1} g(x, y), \\
u_{k+1}(x, y)=-L_{x}^{-1} L_{y} u_{k}(x, y)-L_{x}^{-1} R\left(u_{k}(x, y)\right)-L_{x}^{-1} A_{k}, k \geq 0
\end{gathered}
$$

Using the algorithms described before for calculating $A_{n}$ for the nonlinear term $F(u)$.

The first few components can be identified by

$$
\begin{gathered}
u_{0}(x, y)=\varnothing_{0}-L_{x}^{-1} g(x, y), \\
u_{1}(x, y)=-L_{x}^{-1} L_{y} u_{0}(x, y)-L_{x}^{-1} R\left(u_{0}(x, y)\right)-L_{x}^{-1} A_{0}, \\
u_{2}(x, y)=-L_{x}^{-1} L_{y} u_{1}(x, y)-L_{x}^{-1} R\left(u_{1}(x, y)\right)-L_{x}^{-1} A_{1}, \\
u_{3}(x, y)=-L_{x}^{-1} L_{y} u_{2}(x, y)-L_{x}^{-1} R\left(u_{2}(x, y)\right)-L_{x}^{-1} A_{2}, \\
u_{4}(x, y)=-L_{x}^{-1} L_{y} u_{3}(x, y)-L_{x}^{-1} R\left(u_{3}(x, y)\right)-L_{x}^{-1} A_{3},
\end{gathered}
$$

where each components can be determined by using the preceding component. Having calculated the components $u_{n}(x, y)$.

\section{Numerical Examples}

In this section, we solve some examples, and we can compare the numerical results with the exact solution.

Example 1. Consider the nonlinear partial differential equation 


$$
u_{t}+u u_{x}=0, u(x, 0)=x, t>0,
$$

the exact Solution $u(x)=\frac{x}{1+t},|t|<1$.

Applying the Adomian decomposition method using Maple18.

Example 2. Consider the nonlinear partial differential equation.

$$
u_{t}+u u_{x}=1+t \cdot \cos x+\sin x \cos x, u(x, 0)=\sin x
$$

the exact Solution $u(x)=t+\sin x$.

Applying the Adomian Decomposition Method using Maple18.

Example 3. Consider the nonlinear partial differential equation

$$
u_{t}=x^{2}+\frac{1}{4} u_{x}^{2}, u(x, 0)=0
$$

the exact Solution $u(x)=x^{2} \tan t$.

Applying the Adomian Decomposition Method using Maple18.

Example 4. Consider the nonlinear partial differential equation

$$
u_{t}+\frac{1}{36} x u_{x x}^{2}=x^{3}, u(x, 0)=0 \text {, }
$$

the exact Solution $u(x, t)=x^{3} \tanh t$.

Applying the Adomian Decomposition Method using Maple.

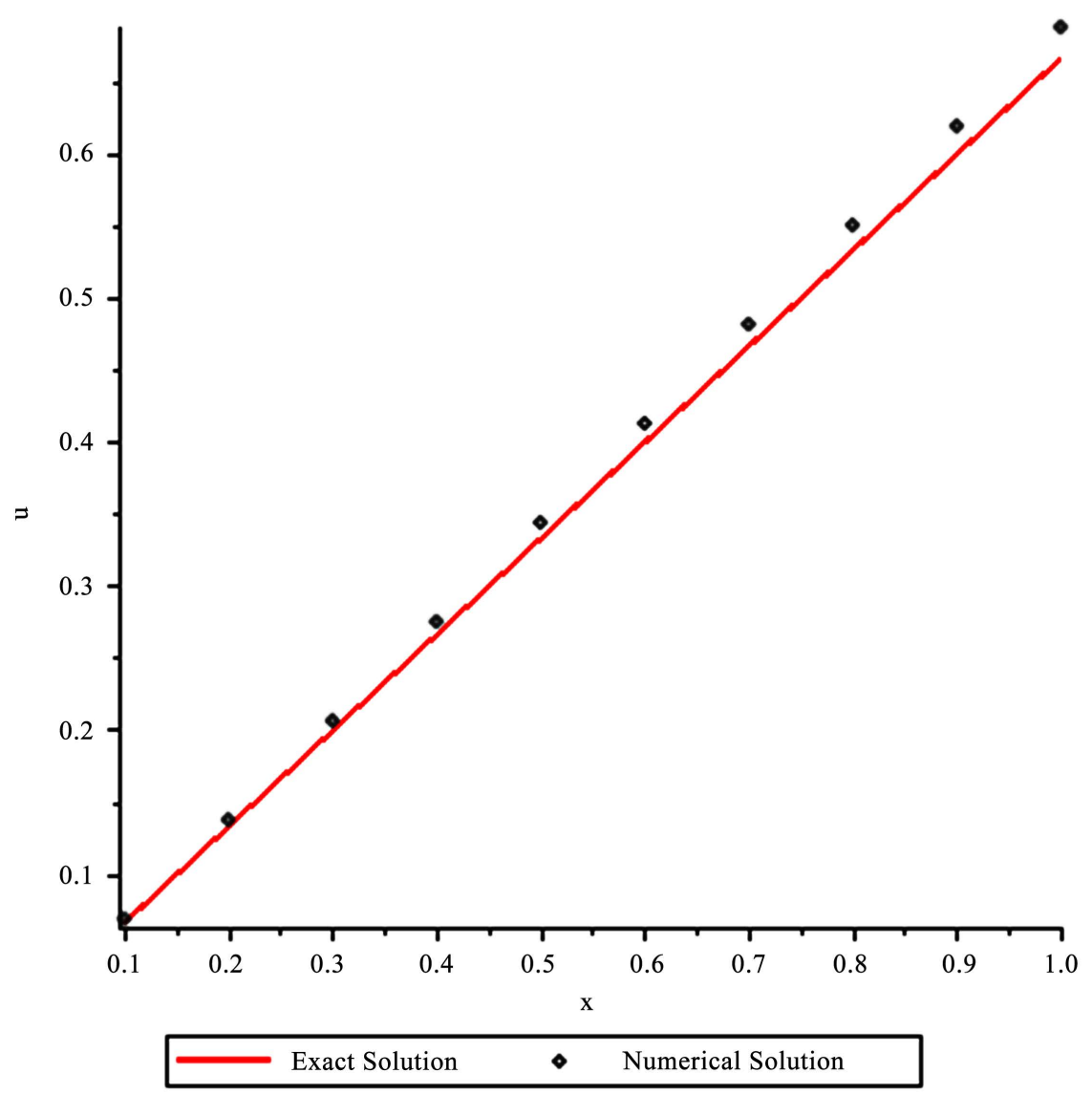

Figure 1. Plot of the solutions of nonlinear partial differential equation for example 1. 


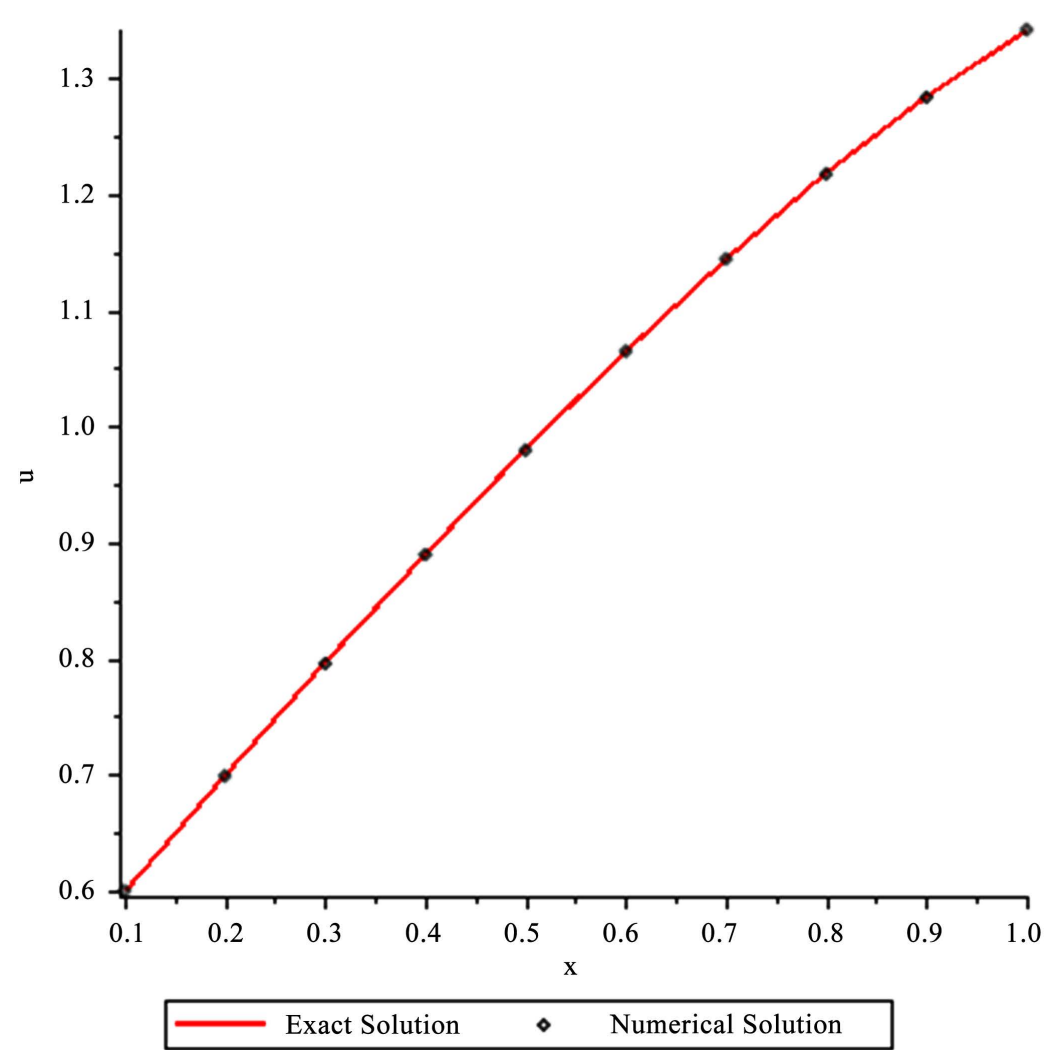

Figure 2. Plot of the solutions of nonlinear partial differential equation for example 2.

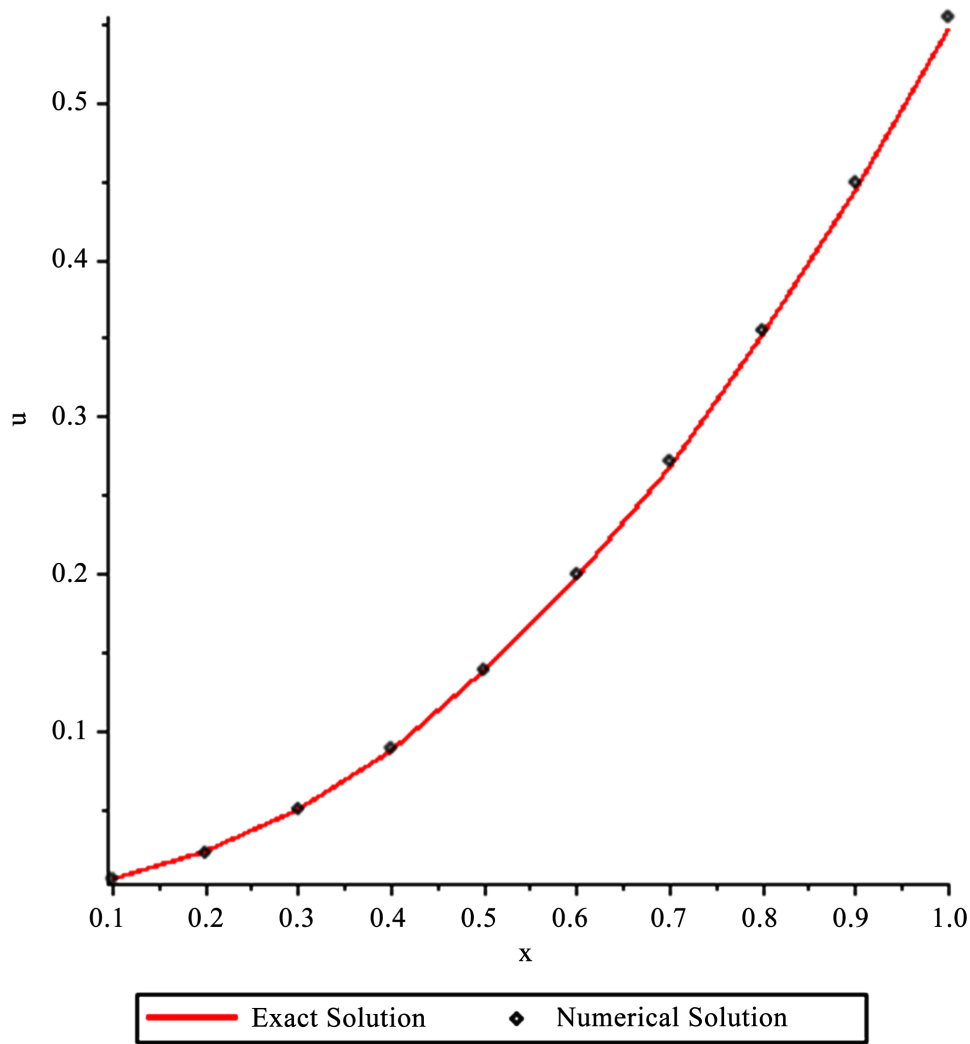

Figure 3. Plot of the solutions of nonlinear partial differential equation for example 3. 
Table 1. Approximation solution and exact solution of nonlinear partial differential equation for example 1 .

\begin{tabular}{cccc}
\hline$x$ & $u$ & Exact $2=\frac{x}{1+t}$ & Error $=\mid$ Exact $1-u \mid$ \\
\hline 0.10000 & 0.06666667 & 0.0687500 & 0.0020833 \\
0.20000 & 0.1333333 & 0.1375000 & 0.0041667 \\
0.30000 & 0.2000000 & 0.2062500 & 0.0062500 \\
0.40000 & 0.26666667 & 0.2750000 & 0.0083333 \\
0.50000 & 0.3333333 & 0.3437500 & 0.0104167 \\
0.60000 & 0.4000000 & 0.4125000 & 0.0125000 \\
0.70000 & 0.46666667 & 0.4812500 & 0.0145833 \\
0.80000 & 0.5333333 & 0.5500000 & 0.0166667 \\
0.90000 & 0.6000000 & 0.6187500 & 0.0187500 \\
1.00000 & 0.6666667 & 0.6875000 & 0.0208333 \\
\hline
\end{tabular}

Table 2. Approximation solution and exact solution of nonlinear partial differential equation for example 2 .

\begin{tabular}{cccc}
\hline$x$ & $u$ & Exact $2=\sin (x)$ & Error $=\mid$ Exact $2-u \mid$ \\
\hline 0.10000 & 0.5998334 & 0.5998334 & 0.0000000 \\
0.20000 & 0.6986693 & 0.6986693 & 0.0000000 \\
0.30000 & 0.7955202 & 0.7955202 & 0.0000000 \\
0.40000 & 0.8894183 & 0.8894187 & 0.0000003 \\
0.50000 & 0.9794255 & 0.9794271 & 0.0000015 \\
0.60000 & 1.0646425 & 1.0646480 & 0.0000055 \\
0.70000 & 1.1442177 & 1.1442339 & 0.0000162 \\
0.80000 & 1.2173561 & 1.2173973 & 0.0000412 \\
0.90000 & 1.2833269 & 1.2834208 & 0.0000938 \\
1.00000 & 1.3414710 & 1.3416667 & 0.0001957 \\
\hline
\end{tabular}

Table 3. Approximation solution and exact solution of Volterra Fredholm integral equations for example 3.

\begin{tabular}{cccc}
\hline$x$ & $u$ & Exact $3=x^{2}$ tan $t$ & Error $=\mid$ Exact $3-u \mid$ \\
\hline 0.10000 & 0.0054630 & 0.0055459 & 0.0000829 \\
0.20000 & 0.0218521 & 0.0221835 & 0.0003314 \\
0.30000 & 0.0491672 & 0.0499129 & 0.0007457 \\
0.40000 & 0.0874084 & 0.0887341 & 0.0013257 \\
0.50000 & 0.1365756 & 0.1386471 & 0.0020715 \\
0.60000 & 0.1966689 & 0.1996518 & 0.0029829 \\
0.70000 & 0.2676882 & 0.2717483 & 0.0040600 \\
0.80000 & 0.3496336 & 0.3549365 & 0.0053029 \\
0.90000 & 0.4425050 & 0.4492165 & 0.0067115 \\
1.00000 & 0.5463025 & 0.5545883 & 0.0082858 \\
\hline
\end{tabular}




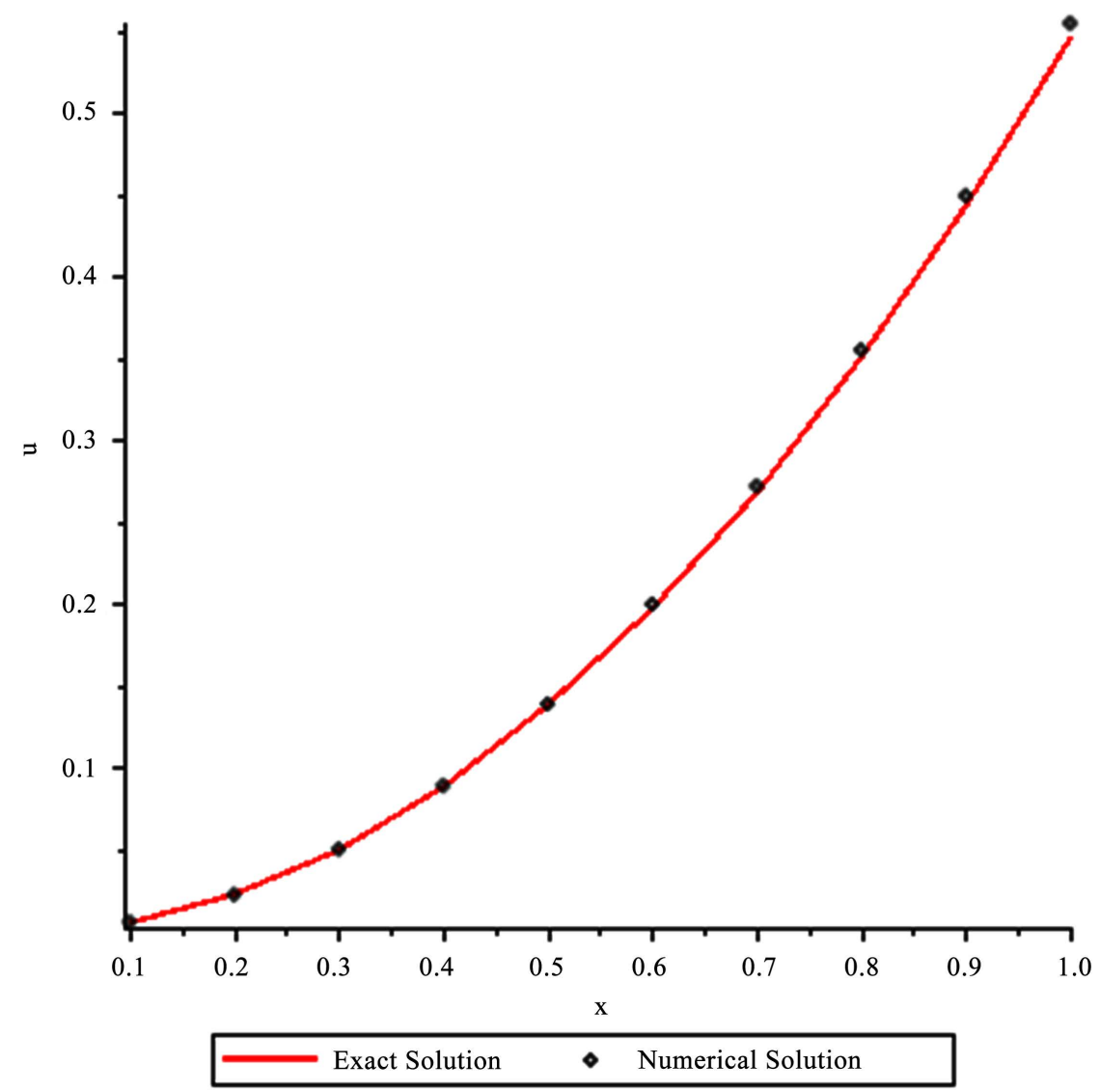

Figure 4. Plot of the solutions of of nonlinear partial differential equation for example 4.

Table 4. Approximation solution and exact solution of nonlinear partial differential equation for example 4 .

\begin{tabular}{cccc}
\hline$x$ & $u$ & Exact $4=x^{3} \tanh t$ & Error $=\mid$ Exact $4-u \mid$ \\
\hline 0.10000 & 0.0004621 & 0.0004704 & 0.0000083 \\
0.20000 & 0.0036969 & 0.0037633 & 0.0000664 \\
0.30000 & 0.0124772 & 0.0127011 & 0.0002240 \\
0.40000 & 0.0295755 & 0.0301063 & 0.0005309 \\
0.50000 & 0.0577646 & 0.0588015 & 0.0010368 \\
0.60000 & 0.0998173 & 0.1016089 & 0.0017916 \\
0.70000 & 0.1585062 & 0.1613512 & 0.0028450 \\
0.80000 & 0.2366040 & 0.2408508 & 0.0042468 \\
0.90000 & 0.3368834 & 0.3429301 & 0.0060467 \\
1.00000 & 0.4621172 & 0.4704117 & 0.0082945
\end{tabular}

\section{Conclusion}

In this paper, the Adomian decomposition method is applied to solve the nonlinear partial differential equation using Maple18 software. Results were obtained by tables and drawing with figures. The exact solution and numerical solution 
are shown in Tables 1-4 and Figures 1-4. By comparing the numerical results, we find that the numerical solution is widely applied to the precise solution, which proves the efficiency of the method used and the ability to obtain the numerical solution corresponding to the precise solution easily and conveniently using Maple 18 software. Moreover, high accuracy of the results obtained.

\section{Acknowledgements}

This project was funded by the Deanship of Scientific Research (DSR), King Abdulaziz University. The authors, therefore, acknowledge with thanks DSR technical and financial support.

\section{Conflicts of Interest}

The authors declare no conflicts of interest regarding the publication of this paper.

\section{References}

[1] Wazwa, A.-M. (1999) A Reliable Modification of Adomain Decomposition Method. Applied Mathematics and Computation, 102, 77-86. https://doi.org/10.1016/S0096-3003(98)10024-3

[2] Wazwa, A.-M. and El-Sayed, S.A. (2001) Anew Modification of the Adomain Decomposition Method for Linear and Nonlinear Operators. Applied Mathematics and Computation, 122, 393-405. https://doi.org/10.1016/S0096-3003(00)00060-6

[3] Vadasz, P. and Olek, S. (2000) Convergence and Accuracy of Adomian's Decomposition Method for the Solution of Lorenz Equations. International Journal of Heat and Mass Transfer, 43, 1715-1734. https://doi.org/10.1016/S0017-9310(99)00260-4

[4] El-Tawil, M.A., Bahnasawi, A.A. and Abdel-Naby, A. (2004) Solving Riccati Differential Equation Using Adomian's Decomposition Method. Applied Mathematics and Computation, 157, 503-514. https://doi.org/10.1016/j.amc.2003.08.049

[5] Ghosh, S., Roy, A. and Roy, D. (2007) An Adaptation of Adomian Decomposition for Numeric-Analytic Integration of Strongly Nonlinear and Chaotic Oscillators. Computer Methods in Applied Mechanics and Engineering, 196, 1133-1153. https://doi.org/10.1016/j.cma.2006.08.010

[6] Ebadi, G. and Rashedi, S. (2010) The Extended Adomian Decomposition Method for Fourth Order Boundary Value Problems. Acta Universitatis Apulensis, 22, 65-78.

[7] Tatari, M. and Dehghan, M. (2006) The Use of the Adomian Decomposition Method for Solving Multipoint Boundary Value Problems. Physica Scripta, 73, 672-676. https://doi.org/10.1088/0031-8949/73/6/023

[8] Azreg-Ainou, M. (2009) A Developed New Algorithm for Evaluating Adomian Polynomials. Computer Modeling in Engineering \& Sciences, 42, 1-18.

[9] Duan, J.S. (2010) An Efficient Algorithm for the Multivariable Adomian Polynomials. Applied Mathematics and Computation, 217, 2456-2467.

https://doi.org/10.1016/j.amc.2010.07.046

[10] Duan, J.S. (2011) Convenient Analytic Recurrence Algorithms for the Adomian Polynomials. Applied Mathematics and Computation, 217, 6337-6348. https://doi.org/10.1016/j.amc.2011.01.007

[11] Duan, J.S., Rach, R., Baleanu, D. and Wazwaz, A.M. (2012) A Review of the Ado- 
mian Decomposition Method and Its Applications to Fractional Differential Equations. Commun. Frac. Calc., 3, 73-99.

[12] Rach, R. (2012) A Bibliography of the Theory and Applications of the Adomian Decomposition Method, 1961-2011. Kybernetes, 41, 1087-1148.

https://doi.org/10.1108/k.2012.06741gaa.008

[13] Abdul-Majid, W. (2011) Linear and Nonlinear Integral Equations. Higher Education Press, Beijing.

[14] Linz, P. (1985) Analytical and Numerical Methods for Volterra Equations (Studies in Applied Mathematics, Series Number 7). SIAM, Philadelphia.

[15] Doddrell, D.M., Forbes, L.K. and Crozier, S. (1997) Calculating Current Densities and Fields Produced by Shielded Magnetic Resonance Imaging Probes. SIAM Journal on Applied Mathematics, 57, 401-425.

https://doi.org/10.1137/S0036139995283110

[16] Dalal, A.M., Amani, Z.B. and Badreeh, M.G. (2014) Numerical Solution of Volterra Integral Equation of Second Kind Using Implicit Trapezoidal. Journal of Advances in Mathematics, 8, 1540-1553.

[17] Dalal, A.M. (2014) Adomian Decomposition Method of Fredholm Integral Equation of the Second Kind Using Maple. Journal of Advances in Mathematics, 9, 1868-1875.

[18] Dalal, A.M. (2014) Application of Adomian Decomposition Method for Solving of Fredholm Integral Equation of the Second Kind. European Journal of Science and Engineering, 9, 1-9.

[19] Dalal, A.M. (2016) Adomian Decomposition Method for Solving of Fredholm Integral Equation of the Second Kind Using Matlab. International Journal of GEOMATE, 11, 2830-2833.

[20] Maturi, D.A. and Malaikah, H. (2018) Numerical Solution of System of Three Nonlinear Volterra Integral Equation Using Implicit Trapezoidal. Journal of Mathematics Research, 10, 44-58. https://doi.org/10.5539/jmr.v10n1p44

[21] Maturi, D.A. (2019) The Modified Decomposition Method for Solving Volterra Integral Equation of the Second Kind Using Maple. International Journal of GEOMATE, 17, 23-28. https://doi.org/10.21660/2019.62.4590

[22] Malaikah, H.H. (2020) The Adomian Decomposition Method for Solving Volterra-Fredholm Integral Equation Using Maple. Applied Mathematics, 11, 779-787. https://doi.org/10.4236/am.2020.118052 